\title{
Alkyl-1H-benzo[d]imidazole synthesis from alkyl bromides and 1,2-benzenediamines by a mild domino reaction
}

\section{Research article}

\section{George Brătulescu}

Universitatea din Craiova, Facultatea de Ştiințe, Departamentul de Chimie, Calea București 107i, Craiova

*E-mail: george.bratulescu@edu.ucv.ro

Received: 03.09.2021 / Accepted: 12.10.2021 / Published: 27.12.2021

\begin{abstract}
A method was implemented for synthesis of 2-alkyl-1H-benzo[d]imidazole derivatives in dry medium. Starting reagents, 1,2-diaminobenzene derivatives and primary alkyl halides using pyridine $N$-oxide is converted into benzimidazoles. The protocol does not use catalyst and solvent. Simplicity and easy work-up are the important advantages of the method.
\end{abstract}

Keywords: heterogeneous medium; benzimidazole; alkyl halides; pyridine $N$-oxide; domino reaction.

\section{INTRODUCTION}

The benzimidazole core can be designated "Master Key" as it is an essential skeleton in many natural and synthetic compounds used by pharmaceutical and medicinal chemistry [1,2].

DOI: 10.52846/AUCCHEM.2021.2.01 
Biological activity exhibits only benzimidazoles substituted in 1, 2 and/or 5(or 6) nucleus positions [3].

Benzimidazole derivatives are considered an important heterocyclic motif that features a wide range of applications as therapeutic agents including antitumors [4], antifungals [5], antivirals [6], antidepressants [7], antihypertensives [8], anti-convulsants [9], antiHIVs [10], antibacterials [11], proton pump inhibitors [12, 13], antidiabetics and antiasthmatics [14].

The most commonly used method of synthesizing 2-substituded benzimidazoles involves the condensation reaction of 1,4benzenediamines and carboxylic acids or their derivatives with the help of strong inorganic acid catalysts [15, 16]. 2-Alkylbenzimidazoles can be prepared via condensation reaction of o-phenylenediamines with aromatic aldehydes over nano- $\mathrm{Ni}(\mathrm{II}) / \mathrm{Y}$ zeolite as a heterogeneous catalyst [17].

The reduction of 2-nitroanilines with sodium hydride followed by alkylation with organic halides [18] or reduction with stannous chloride followed by reaction with an organic acid anhydride [19] also provides 2alkylbenzimidazoles. Thermal decomposition of 2-azidobenzenamines in the presence of an aldehyde produces 2-alkyl-substituted benzimidazoles [20]. Green methods involving microwaves have also been used to synthesize 2-alkylbenzimidazole derivatives [21, 22].

Previously, we developed a proceeding for obtaining 2arylbenzimidazoles in heterogeneous medium from benzylic halides and o-phenylenediamines [23, 24]. In this line, starting from primary alkyl halides and 1,2-benzenediamine derivatives, we have extended the new method to obtain 2-alkyl-substituted benzimidazoles as well.

\section{MATERIALS AND METHODS}

\subsection{Materials}

All reagents, 1, 2-benzenediamines, alkyl bromides, and pyridine- $\mathrm{N}$-oxide are commercial compounds from Fluka or Aldrich. 
The reagents used in the synthesis have analytical purity and are used as such.

\subsection{Apparatus}

Infrared spectra were recorded using an Alpha Bruker Optics spectrometer in the range of $600-4000 \mathrm{~cm}^{-1}$ at ambient temperature. The melting points were determined on a Gallenkamp digital melting point apparatus. The purity of the compounds and their characterization was done by thin layer chromatography on silica gel glass plates (TLC, $\mathrm{MeOH}-\mathrm{DCM}$ ). The structure of the products was confirmed by comparing their physical properties (melting temperatures, IR absorption bands) with those of the known compounds from the literature.

\subsection{Methods}

General procedure for the synthesis of 2-alkyl-substituted-1H-benzo [d] imidazole derivatives (1-5)

In a round bottom flask fitted with a reflux bulb condenser were added alkyl bromide (6 mmol), 1,2-benzenediamines derivative (6 $\mathrm{mmol})$, and pyridine- $\mathrm{N}$-oxide $(21 \mathrm{mmol})$. The mixture of reactants was heated to selected temperature for required time. After completion of reaction (TLC, $\mathrm{MeOH}-\mathrm{DCM}$ ) the resulting mixture was washed with dilute $\mathrm{NaOH}$ and filtered off. The obtained precipitate was recrystallized from EtOH- $\mathrm{H}_{2} \mathrm{O}$ to isolate 2-alkyl-substituted-1Hbenzo[d]imidazole compound (Table II).

2-Methyl-1H-benzo[d]imidazole (1) [25]. Yield: $72 \%$; mp. $172-173^{\circ} \mathrm{C}$; Molecular formula $\mathrm{C}_{8} \mathrm{H}_{8} \mathrm{~N}_{2}$; IR: 3178s, 2544s, 1668m, 1570m, 1490vs, $1464 \mathrm{~m}, 1440 \mathrm{~m}, 1362 \mathrm{vs}, 1220 \mathrm{~m}, 1044 \mathrm{vs}, 1004 \mathrm{~s}, 897 \mathrm{vs} \mathrm{cm}^{-1}$;

2-Ethyl-1H-benzo[d]midazole (2) [26]. Yield: $70 \%$; $\mathrm{mp} \cdot 174-175^{\circ} \mathrm{C}$; Molecular formula $\mathrm{C}_{9} \mathrm{H}_{10} \mathrm{~N}_{2}$; IR: 3085s, 2640s, $1550 \mathrm{~m}, 1460 \mathrm{vs}, 1410 \mathrm{vs,}$ 1271vs, 1043s, 966m, 748vs cm${ }^{-1}$; 
2-Vinyl-1H-benzo[d]imidazole (3) [27]. Yield: 84\%; mp. 152-153 ${ }^{\circ} \mathrm{C}$; Molecular formula $\mathrm{C}_{9} \mathrm{H}_{8} \mathrm{~N}_{2}$; IR: 3221s, 2945s, 2631m, 1649m, 1450vs, 1262 vs, $1037 \mathrm{~m}, 768$ vs cm$^{-1}$;

2-Ethynyl-1H-benzo[d]imidazole (4) [28]. Yield: $86 \%$; mp. $183-184^{\circ} \mathrm{C}$; Molecular formula $\mathrm{C}_{9} \mathrm{H}_{6} \mathrm{~N}_{2}$; IR: 3198m, 2910s, 2050w, 1580m, 1442 vs, 1245vs, 752. cm ${ }^{-1}$;

2-(1-Propen-1-yl)-1H-benzo[d]imidazole (5) [29]. Yield: 88\%; mp. 192$193^{\circ} \mathrm{C}$; Molecular formula $\mathrm{C}_{10} \mathrm{H}_{10} \mathrm{~N}_{2}$; IR: 3219s, 2939s, $2627 \mathrm{w}, 1640 \mathrm{~m}$, $1447 \mathrm{~s}, 1258 \mathrm{vs}, 1031 \mathrm{w}, 780 \mathrm{vs} \mathrm{cm}^{-1}$;

\section{RESULTS AND DISCUSSION}

We developed a protocol to obtain benzimidazoles by a one-pot two-component domino reaction. An initiator as pyridine $\mathrm{N}$-oxide was used. The synthesis of 2-alky-1H-benzo[d]imidazoles occurs in dry medium without any catalyst (Scheme 1).

$\mathrm{R}^{1}: \mathrm{Me}, \mathrm{Et}$, vinyl, ethynyl, 1-propenyl, tert-Bu; $\mathrm{R}^{2}: \mathrm{H}, \mathrm{Me}, \mathrm{NO}_{2}$

Scheme 1. 2-Alkyl- $1 H$-benzo [d] imidazole synthesis initiated by pyridine $N$ oxide

Optimal conditions of synthesis were determined to obtain 2-(1propen-1-yl)-1H-benzo[d]imidazole from crotyl bromide and 1,2benzenediamine. For this, several determinations were made by modifying the reaction parameters such as the molar ratio of reactants, temperature and time (Table 1). 
Table 1. Finding of the optimal conditions for the synthesis of 2-(1-propen-1yl)-1H-benzo[d]imidazole.

\begin{tabular}{|c|c|c|c|c|c|c|}
\hline \multirow[t]{2}{*}{ Entry } & \multicolumn{3}{|c|}{ Molar ratio } & \multirow{2}{*}{$\begin{array}{c}\text { Time } \\
(\mathrm{h})\end{array}$} & \multirow{2}{*}{$\begin{array}{c}\mathrm{T} \\
\left({ }^{\circ} \mathrm{C}\right)\end{array}$} & \multirow{2}{*}{$\begin{array}{c}\text { Yield } \\
(\%)\end{array}$} \\
\hline & $\mathrm{H}_{3} \mathrm{CCH}=\mathrm{CHCH}_{2} \mathrm{Br}$ & $1,2-\left(\mathrm{NH}_{2}\right)_{2} \mathrm{C}_{6} \mathrm{H}_{4}$ & $\mathrm{PyO}$ & & & \\
\hline 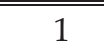 & 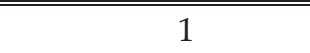 & 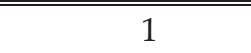 & 1 & 3 & 90 & 38 \\
\hline 2 & 1 & 2 & 1 & 4 & 90 & 46 \\
\hline 3 & 1 & 1 & 1.5 & 2 & 95 & 60 \\
\hline 4 & 1 & 1 & 2.5 & 4 & 95 & 85 \\
\hline 5 & 1.5 & 1 & 1 & 3.5 & 95 & 65 \\
\hline 6 & 1 & 1 & 2 & 4 & 95 & 81 \\
\hline 7 & 1 & 1 & 2 & 2.5 & 100 & 62 \\
\hline 8 & 1 & 1 & 3 & 3 & 95 & 86 \\
\hline 9 & 1 & 1 & 3.5 & 4 & 95 & 88 \\
\hline 10 & 1 & 1 & 4 & 4 & 95 & 88 \\
\hline 11 & 1.5 & 1 & 2.5 & 4.5 & 100 & 71 \\
\hline
\end{tabular}

The halogenated compound and the aromatic amine are in equimolar amount, but an excess of $75 \%$ pyridine $N$-oxide is required. The synthesis occurs at $95^{\circ} \mathrm{C}$ for $4 \mathrm{~h}$. Under these conditions, 2-(1propen-1-yl)- $1 H$-benzo[d]imidazole is obtained in a yield of $88 \%$. With this set of reaction conditions, a series of 2-alkylbenzimidazoles were synthesized (Table 2).

We suppose that pyridine- $N$-oxide transforms alkyl halide into aldehyde (I) by Kornblum oxidation (Scheme 2) [23]. The resulting aliphatic aldehyde (I) forms a Schiff base (II) with the 1,2benzenediamine derivative. An intramolecular cycloaddition of Schiff (II) base generates 2-alkyl-2,3-dihydro- $1 H$-benzo[d]imidazole (III) which was oxidized to 2-alkyl-1H-benzo[d]imidazole (IV) by another pyridine $N$-oxide molecule. 
Table 2. Synthesis of 2-alkyl-1H-benzo[d]imidazoles by a domino reaction.

\begin{tabular}{ccccc} 
& & & \\
\hline Entry & $\mathrm{R}^{1}$ & $\mathrm{R}^{2}$ & $\begin{array}{c}\text { Time } \\
(\mathrm{h})\end{array}$ & $\begin{array}{c}\text { Yield } \\
(\%)\end{array}$ \\
\hline \hline 1 & $\mathrm{Me}$ & $\mathrm{H}$ & 5 & 72 \\
2 & $\mathrm{Et}$ & $\mathrm{H}$ & 5.5 & 70 \\
3 & $\mathrm{C}_{2} \mathrm{H}_{3}$ & $\mathrm{H}$ & 4 & 84 \\
4 & $\mathrm{C}_{2} \mathrm{H}$ & $\mathrm{H}$ & 4.5 & 86 \\
5 & 1-Propen-1-yl & $\mathrm{H}$ & 4 & 88 \\
\hline \hline
\end{tabular}
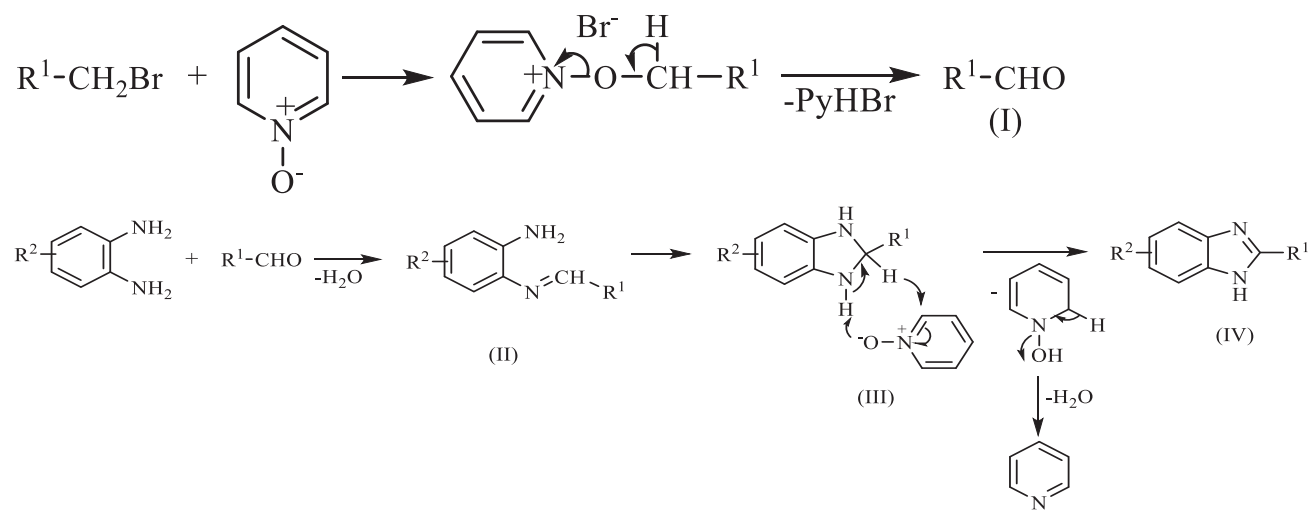

Scheme 2. Presumed reaction mechanism for obtaining 2-alkyl-1Hbenzo[d]imidazoles

\section{CONCLUSIONS}

A smooth procedure that allows easy synthesis of 2-alkylsubstituted- $1 H$-benzo[d]imidazoles from 1,2-benzenediamines, pyridine- $N$ oxide and a variety of alkyl bromides was developed. The novel one-pot domino sequence uses thermal heating conditions and does not require catalyst and solvent. The method allows the obtaining of the alkyl benzimidazoles in good yields, but the reaction time is longer than in the case of obtaining the aryl benzimidazoles [23, 24]. The main advantages of the procedure are mild conditions, the operational simplicity, easy isolation of alkyl benzimidazole derivatives from reaction medium, and also simple work-up. 


\section{REFERENCES}

1. R. S. Keri, A. Hiremathad, S. Budagumpi, B. M. Nagaraja, Chem. Biol. Drug. Des., 86 (2015) 799.

2. M. Wang, X. Han, Z. Zhou, Expert Opin. Ther. Pat., 25 (2015) 595.

3. Y. Bansal, O. Silakari, Bioorganic Med. Chem., 20 (2012) 6208.

4. K. J. Soderlind, B. Gorodetsky, A. K. Singh, N. R. Bachur, G. G. Miller, J. W. Lown, Anticancer Drug Des., 14 (1999) 19.

5. K. G. Desai, K. R. Desai, Bioorg. Med. Chem., 14 (2006) 8271.

6. B. L. Townsend, R. V. Devivar, S. R. Turk, M. R. Nassiri, J. C. Drach, J. Med. Chem., 38 (1996) 4098.

7. B. Mathew, J. Suresh, S. Anbazhagan, J. Saudi. Chem. Soc., 20 (2015) 132.

8. A. Jain, R. Sharma, S. C. Chaturvedi, Med. Chem. Res., 22, (2013) 4622.

9. N. Siddiqui, D. Pathak, M. S. Alam, R. Ali, B. Azad, Acta Pol. Pharm., 69 (2012) 53.

10. G. R. Liu, J. Liu, Q. Pan, Z. B. Song, F. L. Luo, S. R. Wang, X. L. Zhang, X. Zhou, Chem. Biodivers, 12 (2009) 2200.

11. E. I. Elnima, M. U. Zubair, A. A. Al-Badr, Antimicrob. Agents Chemother., 19 (1981) 29.

12. M. Uchida, S. Morita, M. Chihiro, T. Kanbe, K. Yamasaki, Y. Yabuuchi, K. Nakagawa, Chem. Pharm. Bull., 37 (1989) 1517.

13. M. Baumann, I. R. Baxendale, S. V. Ley, N. Nikbin, Beilstein J. Org. Chem., 7 (2011) 442.

14. V. K. Ramanatham, S. Dashrath Vaidya, B. Venkata Siva Kumar, U. Bhise, S. Bhirud, U. Chandrakant Mashelkar, Eur. J. Med. Chem., 43 (2008) 986.

15. F. A. S. Alasmary, A. M. Snelling, M. E. Zain, A. M. Alafeefy, A. S. Awaad, N. Karodia, Molecules, 20 (2015) 15206.

16. S. S. Panda, R. Malikb, S. C Jain, Curr. Org. Chem., 16 (2012)1905.

17. A.Mobinikhaledi, M. Zendehdel, F. Goudarzi, G.R. Bardajee, Synth. React. Inorg. Met. Org. Chem., 46 (2015)1526.

18. J. M. Gardiner, C. R. Loyns, C. H. Schwalbe, G. C. Barrett, P. R. Lowe, Tetrahedron, 51 (1995) 4101.

19. J. M. Gardiner and C. R. Loyns, Synth. Commun., 25 (1995) 819.

20. J. M. Wallace, B. C. G. Soderberg, J. W. Hubbard, Synth. Commun., 36 (2006) 3425.

21. C. Mukhopadhyay, S. Ghosh, S. Sengupta, S. De, RSC Adv., 1 (2011) 1033.

22. A. Saberi, K. S. Rangappa, Synth. React. Inorg. Met. Org. Chem., 39 (2009) 425.

23. G. Bratulescu, Synth. Commun, 47 (2017) 811.

24. G. Bratulescu, ARKIVOC, IV (2017) 95.

25. S. F. Hojati, B. Maleki, Z. Beykzadeh, Monatsh. Chem., 142 (2011) 87.

26. Z. H. Zhang, , J. J. Li, Y. Z. Gao, Y. H. Liu, J. Heterocyclic Chem., 44 (2007) 1509.

27. J. W. Hubbard, A. M. Piegols, B. C. G. Söderberg, Tetrahedron, 63 (2007) 7077.

28. A. Zubenko, I.I. Popov, A.M. Simonov. Khim., Geterotsikl. Soedin., 8 (1978), 1111.

29. M. T. Le Bris, Bull. Soc. Chim. Fr., 9 (1967)3411. 